\title{
The Relationship Between the Supply of Primary Care Physicians and Measures of Breast Health Service Use
}

\author{
Janis Barry, $\mathrm{PhD}$
}

\begin{abstract}
Background: To investigate whether women are more likely to report receipt of a mammography recommendation from a doctor or mammography use if they reside in primary care service areas (PCSAs) having a greater number of clinically active primary care physicians.

Materials and Methods: The analysis used a nationally representative sample of women, aged 40 years and above ( $n=10,706$ unweighted respondents), extracted from the 2005 National Health Interview Survey. The restricted geocoded addresses of the respondents were linked to PCSA data on physician density at a secure research data center. Multivariable logistic regression was used to determine whether, after adjustment, specific measures of primary care providers (e.g., the number of obstetricians and gynecologists [Ob-GyNs] per 10,000 population) were associated with either recommendation receipt or mammography use.

Results: After adjusting for other factors, a one-unit increase in the PCSA number of Ob-GyNs per 10,000 population increased the odds of mammography recommendation receipt by $9 \%$ and the odds of mammography use by $9 \%$. The ratio of international medical graduate Ob-GyNs to US-trained Ob-GyNs in a PCSA was negatively associated with mammography use.

Conclusion: The results from this nationwide study underscore the importance of using physician density measures estimated from within bounded medical markets, where women reside and actually seek preventive breast health services. Results support the hypothesis that PCSA physician supply is independently associated with both mammography recommendation receipt and mammography utilization.
\end{abstract}

Keywords: cancer, breast health, preventive medicine, primary care

\section{Introduction}

$\mathbf{I}_{\mathrm{i},}$ N THE UNITED STATES, a primary care physician referral is usually needed for a woman to obtain a mammogram. Women report that receiving a doctor's recommendation is critical to their decision to utilize screening mammography. ${ }^{1-5}$ A woman's decision to utilize mammography is determined by factors separate from, but related to, getting a physician's recommendation. ${ }^{6-8}$ Differences in recommendation receipt probabilities are associated with individual socioeconomic status, gender of the respondent's provider, and residence in a low-income urban community. ${ }^{9-11}$

Studies have investigated the association between physician density and use of breast cancer care services in the United States, Canada, Germany, and France. ${ }^{12-16}$ Discrepancies in findings among US-based studies may be due to the different levels of geography (i.e., states, county, MSA, census tract) at which physician supply is measured. ${ }^{17-19}$ Additionally, the examination of dissimilar outcomes, including breast cancer survival, stage of breast cancer diagnosis, and mammography screening use, makes comparisons of results across studies difficult. $^{20,21}$

None of the referenced research has considered the impact of physician capacity on the probability of getting a mammography recommendation from a doctor. Since recommendation receipt is usually a precursor to mammography use, I investigate whether variations in the geographic distribution of primary care physicians in the United States have contributed to disparities in recommendation receipt as well as to screening mammography use. I extend the existing

Department of Economics, Fordham University, New York, New York.

(C) Janis Barry, 2017; Published by Mary Ann Liebert, Inc. This article is available under the Creative Commons License CC-BY-NC (http://creativecommons.org/licenses/by-nc/4.0). This license permits non-commercial use, distribution and reproduction in any medium, provided the original work is properly cited. Permission only needs to be obtained for commercial use and can be done via RightsLink. 
research on both recommendation receipt and mammography use by utilizing a nationally representative sample of women from the National Health Interview Survey (NHIS) to examine the impact of physician capacity on these two outcomes. This study represents an advance on prior studies of the association between physician capacity and breast cancer screening use as it applies national primary care physician measures generated from within recognized, bounded healthcare markets called primary care service areas (PCSAs). ${ }^{22}$ It also differentiates primary care obstetricians and gynecologists (Ob-GyNs) from indicators for all other primary care physicians, who reported specialties of family practice, internal medicine, or pediatrics. ${ }^{23}$

\section{Materials and Methods}

Data from the 2005 NHIS and the 2005 Cancer Control Supplement (CCS) to the NHIS were used to construct demographic and health data for respondents. The NHIS is a statistically representative in-person household interview of the US civilian noninstitutionalized population conducted annually and based on a multistage, clustered area probability sample. In 2005, the CCS was administered to the entire adult sample. The response rate for the core NHIS survey in 2005 was $86.5 \%$ and $69 \%$ for the CCS. ${ }^{24}$

Due to my use of restricted geocodes (state, county, and census tract identifiers) associated with the NHIS respondent's address, statistical inspection of the household files of individuals in the NHIS who were linked to PCSA data was examined at a secure premise, the Research Data Center (RDC), at the National Center for Health Statistics (NCHS) in Hyattsville, MD, after approval by an NCHS review board. When the project was undertaken, the 2005 NHIS was the most recent year for which 2005-2006 PCSA physician data were available for analysis.

\section{Sample}

All women aged 40 years and over, reporting their mammography use in the past 2 years at time of interview, are included in the sample. Excluded from the sample were those who reported that their screening mammography was for reasons other than as part of a routine physical examination or who reported being previously diagnosed with cancer.

The choice of the aged 40 years and over subpopulation is consistent with the 2005 recommendations for receiving screening mammography. ${ }^{25}$ In 2009, the US Preventive Services Task Force changed its recommendation for women aged $40-49$ years to get a routine mammography. ${ }^{26}$ Alternatively, the American Cancer Society continued to support the right of women aged 40-44 years to choose screening and advised women aged $45-54$ years to get a mammogram every year. ${ }^{27}$

\section{Dependent variables}

Self-report of a mammography recommendation received from a doctor during the prior year was the first outcome measure tested. The second dependent variable was selfreported mammography use during the previous 2 years. The cross-sectional design of the NHIS means it is impossible to know what number of women who reported they saw a doctor in the past year and a doctor recommended a mammogram did so before reporting their mammography use in the pre- vious 2 years. Therefore, it is likely that the recommendation outcome measure underestimates the actual number of recommendations received by women who reported mammograms in the past 2 years.

\section{PCSA covariates}

Funded by the Health Resources and Service Administration (HRSA), there are 6,542 PCSAs nationally, formulated from the records of Medicare patients and based on their patterns of local primary care use (HRSA Health Workforce). PCSA data from the years 2005 and 2006 were used to construct the physician supply variables. ${ }^{23,28}$ Four unique physician supply measures were calculated and are presented here: (1) the number of clinically active Ob-GyNs in the PCSA divided by the 2006 civilian population and computed as ObGyNs per 10,000 population; (2) the number of clinically active primary care physicians in the PCSA divided by the 2006 civilian population and computed as physician per 10,000 population; and (3) a ratio of the 2005 civilian population to the number of full-time equivalent primary care providers (FTEPCPs) in the PCSA. FTEPCP was defined using the PCSA number of clinically active, nonfederal general and $\mathrm{Ob}$ GyN physicians, summed with the weighted (0.5) number of nonphysicians (i.e., nurse practitioners, physician's assistants, and certified nurse midwives). This variable was coded categorically as $<1,000$ people per FTEPCP, 1,000-3,000 people per FTEPCP, or $>3,000$ people per FTEPCP. The final indicator calculated a ratio of the number of clinically active international medical graduate (IMG) Ob-GyNs relative to the number of US-trained Ob-GyNs located in the PCSA.

Also included were contextual measures related to accessing the healthcare system, including dummy variables indicating PCSAs that had at least $30 \%$ of the population with incomes below 200\% of the 2005 federal poverty level, PCSAs that were high-density (urban core and suburban) regions, the number of unique mammography (mammogram and MRI) providers located inside the PCSA (identified by UPIN with ZIP code centroid), the average travel distance to mammography facilities, and the number of federally qualified health centers within a PCSA. ${ }^{28,29}$

\section{NHIS covariates}

Individual-level covariates were chosen on the basis of their importance as found in previous studies on women's use of breast health services. ${ }^{7}$ Variables included age, nativity, race/ethnicity, education level, family income, employment and marital status, any functional limitations, health insurance coverage, smoking and health status, and reported number of doctor visits in the past year. NHIS controls for geographic areas included the region of residence in the United States (Northeast, South, Midwest, and West).

\section{Statistical analysis}

A final total of 8,677 unweighted observations were used in the adjusted multivariate analyses. The Zip Code Tabulation Area (ZCTA) crosswalk used to merge the PCSA data to the entire adult NHIS showed that $10 \%$ (3,191 unweighted observations) of 31,428 (total unweighted observations) could not be matched due to missing Census block values. That left an unweighted sample of age-appropriate women equal to 
10,706, with a total of 9,682 observations reporting their mammography use. Of these observations, 1,005 were missing PCSA data due to the ZCTA merge. Comparison of excluded respondents who had missing PCSA identifiers with those having identifiers and therefore included in the sample revealed similar rates of mammography use (65\% vs. $66 \%)$.

All reported percentages were weighted and the standard errors for the $95 \%$ confidence intervals calculated by SUDAAN (software version 10.0.1) to account for the 2005 NHIS complex sample design and to obtain sampling weights for generating nationally representative estimates from the linked NHIS and PCSA data. ${ }^{30}$ Respondents with missing values for independent variables included in the estimating equations were excluded from the test results.

Bivariate analyses were initially completed to examine the association between physician density and each of the two outcome measures. Statistical testing for these models was performed using the Wald $F$ test. Due to the large sample size of the NHIS, only independent variables with $p$-values less than 0.05 in the bivariate analysis were permitted inclusion in the multivariate models. Logistic regression analyses of recommended receipt and mammography use were conducted. Parameter estimates from the logistic regressions were converted to odds ratios and predicted margins were generated. The predictive margin for a specific group represents the average predicted response if everyone in the sample had been in that group. These percentages can be interpreted as recommendation receipt and screening use rates after adjustment for all other variables in the model. Predictive margins assist with identifying differences among multiple category variables and also allow for easy comparisons between the unadjusted and adjusted results. Finally, software limitations at the Hyattsville, MD, RDC meant that multilevel models (MLMs) could not be employed. However, questions concerning the efficacy of MLMs when analyzing complex survey data with design weights suggest that methodological and statistical issues have yet to be resolved. ${ }^{19,31,32}$

\section{Results}

Bivariate (unadjusted) analysis of mammography recommendation and mammography use

Sixty-two percent of the age-appropriate sample $(n=9,682)$ reported seeing a doctor in the past year and receiving a mammogram recommendation. Sixty-six percent of this sample reported having used mammography in the past 2 years. The mean number of primary care doctors per 10,000 population across all PCSAs was 7.75 (SD: 319.5), with a minimum value of 0 and a maximum value of 83 . On average, there was one FTEPCP for every 1,558 people across all PCSAs (SEM: 91.38).

Women who lived in PCSAs with more than 3,000 people per FTEPCP were less likely to report a recommendation receipt compared with women who lived in PCSAs with 3,000 or fewer people per FTEPCP. Approximately, 12.1\% (weighted) of respondents in the recommendation receipt sample resided in PCSAs having more than 3,000 people per primary care provider, a ratio approaching the federal health professional shortage area designation. An increase in either the PCSA number of Ob-GyNs or all primary care physicians (per 10,000 population) increased the likelihood of recommendation receipt. An increase in the average distance to the closest mammogram provider and residence in a PCSA characterized by having more than $30 \%$ of the population living in poverty were both associated with a decreased likelihood of receiving a recommendation (Table 1).

An increase in the number of Ob-GyN physicians per 10,000 population was associated with an increased likelihood of mammography screening use, while an increase in the IMG Ob-GyN-to-non-IMG Ob-GyN ratio made it less likely that the respondent reported mammography use. The supply of primary care physicians and the number of mammogram providers in the PCSA were both positively associated with mammogram use (Table 2). Residence in an urban/suburban PCSA increased the odds of mammography use when compared with rural or less densely populated PCSAs. Increased travel distance to the closest mammography provider was associated with a decreased likelihood of reporting a screening mammography.

Other variables showing positive associations with both outcome measures included non-Hispanic white race, US birth, ages 50-64, former smoker, and being married. Lower levels of education and income, fewer doctor visits in the past year, and a lack of health insurance coverage were negatively associated with both recommendation receipt and mammography use (Tables 1 and 2).

There were a number of major differences in the determinants of recommendation receipt when compared with mammography use. Women reporting functional limitations were more likely to have received a recommendation for a mammogram, while women who were limited in any way were less likely to have used mammography in the past 2 years. Region of the country was associated with recommendation receipt, but not mammogram use. Factors associated with mammography use, but not recommendation receipt, included employment and health status, PCSA density, the ratio of IMG Ob-GyNs to non-IMG Ob-GyNs, and the number of mammogram providers in the PCSA. Two additional PCSA factors that were examined, the number of federal health clinics and the number of IMG primary care physicians practicing in the PCSA, were not significantly associated with either of the two outcomes.

\section{Multivariate (adjusted) analyses of recommendation receipt and mammography use}

Table 3 shows that after adjustment for other factors, a one-unit increase in the number of Ob-GyNs in the PCSA increased the likelihood of recommendation receipt by $9 \%$. Distance to closest mammogram provider was negatively associated with recommendation receipt. Fifty-one percent of Asian American and 59\% of non-Hispanic black women reported receiving a mammography recommendation compared with $63 \%$ of non-Hispanic white women. Only $60 \%$ of unmarried women, $52 \%$ of women who lacked health insurance coverage, and $59 \%$ of women living in families with less than $\$ 20,000$ in income reported receiving a recommendation. Alternatively, $60 \%-66 \%$ of women who reported one or more doctor visits, $65 \%$ of women with functional limitations, and $66 \%$ of women aged 50-64 years were more likely to have reported recommendation receipt. Nativity, education level, region, smoking status, and PCSA poverty level were no longer significant in the adjusted results for recommendation receipt. 
Table 1. Selected Primary Care Service Area and Individual Characteristics of Women Aged 40 Years or Older Who Reported Doctor's Recommendation for Mammography Within the Past Year (2005 NHIS)

\begin{tabular}{|c|c|c|c|c|}
\hline Characteristic & $\begin{array}{l}\text { Unweighted } \\
\text { frequency }^{\mathrm{a}}\end{array}$ & Mean & $S D$ & $\begin{array}{l}\text { Crude OR } \\
\left(95 \% C I^{\mathrm{b}}\right)\end{array}$ \\
\hline $\begin{array}{l}\text { Number of Ob-GyNs in PCSA per } 10,000 \text { PCSA population }^{\mathrm{c}, \mathrm{d}} \\
\text { Number of PCMD in PCSA per } 10,000 \text { population }^{\mathrm{c} d} \\
\text { Average distance to closest mammogram in } \text { PCSA }^{\mathrm{c}, \mathrm{d}}\end{array}$ & $\begin{array}{l}7,947 \\
7,947 \\
7,945\end{array}$ & $\begin{array}{l}1.27 \\
7.71 \\
4.47\end{array}$ & $\begin{array}{l}79.7 \\
300 . \\
477\end{array}$ & $\begin{array}{l}1.10(1.04,1.17)^{* *} \\
1.03(1.01,1.04)^{* *} \\
0.99(0.98,1.01)^{*}\end{array}$ \\
\hline & $\begin{array}{l}\text { Unweighted } \\
\text { frequency }\end{array}$ & $\begin{array}{l}\text { Weighted } \\
\% \text { sample }\end{array}$ & $\begin{array}{l}\% \text { Recommend } \\
\text { receipt }\end{array}$ & $\begin{array}{l}\text { Crude OR } \\
(95 \% \text { CI })\end{array}$ \\
\hline $\begin{array}{l}\text { Population to FTEPCP } \\
<1,000 \text { people per PCP } \\
1,000-3,000 \text { people per PCP } \\
>3,000 \text { people per PCP }\end{array}$ & $\begin{array}{r}2,971 \\
4,037 \\
939\end{array}$ & $\begin{array}{l}37.1 \\
50.7 \\
12.1\end{array}$ & $\begin{array}{l}63.4 \\
62.9 \\
56.9\end{array}$ & $\begin{array}{c}1.31(1.12,1.56) \\
1.28(1.06,1.55) \\
1.00 \text { Ref* }^{*}\end{array}$ \\
\hline $\begin{array}{l}\text { PCSA Poverty level } \\
>30 \% \text { of PCSA lives under } 200 \% \text { FPL } \\
<30 \% \text { of PCSA lives under } 200 \% \text { FPL }\end{array}$ & $\begin{array}{l}3,436 \\
4,511\end{array}$ & $\begin{array}{l}47.6 \\
52.3\end{array}$ & $\begin{array}{l}60.0 \\
65.0\end{array}$ & $\begin{array}{r}0.81(0.72,0.91) \\
1.00 \text { Ref**** }\end{array}$ \\
\hline $\begin{array}{l}\text { Race } \\
\text { Non-Hispanic white } \\
\text { Non-Hispanic black } \\
\text { Hispanic } \\
\text { Asian American }\end{array}$ & $\begin{array}{r}6,235 \\
1,221 \\
1,104 \\
197\end{array}$ & $\begin{array}{r}77.6 \\
11.0 \\
8.61 \\
2.66\end{array}$ & $\begin{array}{l}63.9 \\
55.2 \\
56.0 \\
52.3\end{array}$ & $\begin{array}{c}1.62(1.19,2.19) \\
1.12(0.8,1.57) \\
1.16(0.82,1.66) \\
1.00 \mathrm{Ref}^{* * *}\end{array}$ \\
\hline $\begin{array}{l}\text { Marital status } \\
\text { Married } \\
\text { Unmarried (incl. LWP) }\end{array}$ & $\begin{array}{l}4,049 \\
4,793\end{array}$ & $\begin{array}{l}60.1 \\
39.8\end{array}$ & $\begin{array}{l}64.6 \\
58.2\end{array}$ & $\begin{array}{c}1.31(1.18,1.45) \\
1.00 \operatorname{Ref}^{* * *}\end{array}$ \\
\hline $\begin{array}{l}\text { Family income } \\
\quad<20,000 \\
\$ 20,000-34,999 \\
\$ 35,000-54,999 \\
\$ 55,000-74,999 \\
\geq \$ 75,000\end{array}$ & $\begin{array}{r}2,303 \\
1,403 \\
1,267 \\
731 \\
1,507\end{array}$ & $\begin{array}{l}22.9 \\
17.9 \\
18.1 \\
11.9 \\
29.0\end{array}$ & $\begin{array}{l}55.2 \\
58.9 \\
63.7 \\
65.8 \\
69.4\end{array}$ & $\begin{array}{c}0.57(0.49,0.65) \\
0.68(0.57,0.82) \\
0.81(0.69,0.96) \\
0.84(0.68,1.05) \\
1.00 \mathrm{Ref} * * *\end{array}$ \\
\hline $\begin{array}{l}\text { Limitations } \\
\text { Limited in any way } \\
\text { Not limited in any way }\end{array}$ & $\begin{array}{l}4,455 \\
4,401\end{array}$ & $\begin{array}{l}48.2 \\
51.7\end{array}$ & $\begin{array}{l}64.3 \\
60.0\end{array}$ & $\begin{array}{c}1.20(1.09,1.32) \\
1.00 \operatorname{Ref}^{* * * *}\end{array}$ \\
\hline $\begin{array}{l}\text { Health insurance } \\
\text { Not covered } \\
\text { Covered }\end{array}$ & $\begin{array}{r}840 \\
8,016\end{array}$ & $\begin{array}{l}8.51 \\
91.4\end{array}$ & $\begin{array}{l}45.3 \\
63.5\end{array}$ & $\begin{array}{c}0.49(0.41,0.58) \\
1.00 \operatorname{Ref}^{* * *}\end{array}$ \\
\hline $\begin{array}{l}\text { Age group } \\
\quad 40-49 \\
50-64 \\
65+\end{array}$ & $\begin{array}{l}2,727 \\
3,347 \\
2,796\end{array}$ & $\begin{array}{l}33.9 \\
38.6 \\
27.3\end{array}$ & $\begin{array}{l}60.4 \\
65.7 \\
58.9\end{array}$ & $\begin{array}{c}1.06(0.93,1.21) \\
1.33(1.18,1.51) \\
1.00 \text { Ref*** }^{* *}\end{array}$ \\
\hline $\begin{array}{l}\text { Education } \\
\text { Less than H.S. } \\
\text { H.S. grad } \\
\text { Some college } \\
\text { College grad }\end{array}$ & $\begin{array}{l}1,681 \\
2,708 \\
2,389 \\
2,030\end{array}$ & $\begin{array}{l}16.2 \\
31.5 \\
27.5 \\
24.5\end{array}$ & $\begin{array}{l}54.9 \\
61.0 \\
64.2 \\
65.9\end{array}$ & $\begin{array}{c}0.63(0.54,0.74) \\
0.81(0.7,0.94) \\
0.93(0.8,1.07) \\
1.00 \text { Ref*** }\end{array}$ \\
\hline $\begin{array}{l}\text { \#MD visits in last year } \\
\text { None } \\
1 \\
2-5 \\
6+\end{array}$ & $\begin{array}{r}6,61 \\
1,192 \\
3,886 \\
3,080\end{array}$ & $\begin{array}{r}7.1 \\
13.8 \\
44.6 \\
34.2\end{array}$ & $\begin{array}{l}30.3 \\
56.8 \\
65.5 \\
66.6\end{array}$ & $\begin{array}{c}0.22(0.18,0.27) \\
0.66(0.56,0.77) \\
0.95(0.84,1.07) \\
1.00 \mathrm{Ref}_{* * *}\end{array}$ \\
\hline $\begin{array}{l}\text { Nativity } \\
\text { Born in USA } \\
\text { Born outside USA/US territories }\end{array}$ & $\begin{array}{l}7,579 \\
1,282\end{array}$ & $\begin{array}{l}87.3 \\
12.6\end{array}$ & $\begin{array}{l}62.9 \\
56.0\end{array}$ & $\begin{array}{c}1.33(1.15,1.55) \\
1.00 \mathrm{Ref}^{* * *}\end{array}$ \\
\hline $\begin{array}{l}\text { Smoking status } \\
\text { Current } \\
\text { Former } \\
\text { Never }\end{array}$ & $\begin{array}{l}1,427 \\
2,142 \\
5,284\end{array}$ & $\begin{array}{l}15.8 \\
24.1 \\
60.0\end{array}$ & $\begin{array}{l}60.8 \\
66.0 \\
60.9\end{array}$ & $\begin{array}{c}1.00(0.87,1.14) \\
1.25(1.05,1.46) \\
1.00 \mathrm{Ref}^{* *}\end{array}$ \\
\hline $\begin{array}{l}\text { Region } \\
\text { Northeast } \\
\text { Midwest } \\
\text { South } \\
\text { West }\end{array}$ & $\begin{array}{l}1,640 \\
2,129 \\
3,295 \\
1,806\end{array}$ & $\begin{array}{l}19.6 \\
24.9 \\
35.8 \\
19.5\end{array}$ & $\begin{array}{l}66.5 \\
65.9 \\
57.5 \\
60.9\end{array}$ & $\begin{array}{c}1.27(1.07,1.51) \\
1.24(63.8,68.3) \\
0.87(0.75,1.01) \\
1.00 \text { Ref*** }\end{array}$ \\
\hline
\end{tabular}

a The number of respondents by each characteristic may not add up to the total unweighted count due to missing values.

b $p$-Value calculated using Wald $F$ test: $p$-value $*<0.05 ; * * \leq 0.01 ; * * * \leq 0.001$.

${ }^{\mathrm{c}}$ Continuous variables.

${ }^{\mathrm{d}}$ Ob-Gyns = obstetricians and gynecologists, PCMD = primary care physicians. Source: Goodman. ${ }^{23}$

${ }^{\mathrm{e}}$ Source: RTI Spatial Impact Factor Web Data.

${ }^{f}$ FPL, federal poverty line. Source: Goodman and Shipman. ${ }^{28}$

FTEPCP, full-time equivalent primary care provider; NHIS, National Health Interview Survey; Ob-GyNs, obstetrician and gynecologists;

PCSA, primary care service area; LVP, living with partner; OR, odds ratio. 
Table 2. Selected Primary Care Service Area and Individual Characteristics of Women Aged 40 Years or Older Who Reported Mammography Use Within the Past 2 Years (2005 NHIS)

\begin{tabular}{|c|c|c|c|c|}
\hline Characteristic & $\begin{array}{l}\text { Unweighted } \\
\text { frequency }^{\mathrm{a}}\end{array}$ & Mean & $S D$ & $\begin{array}{l}\text { Crude OR } \\
\left(95 \% C I^{\mathrm{b}}\right)\end{array}$ \\
\hline $\begin{array}{l}\text { Number of Ob-GyNs in PCSA per } 10,000 \text { population }^{\mathrm{c}, \mathrm{d}} \\
\text { Number of PCMD in PCSA per } 10,000 \text { population } \\
\text { Ratio of IMG Ob-GyN to US Ob-GyNe,d } \\
\text { Average distance to closest mammogram provider in } \text { PCSA }^{\mathrm{c}, \mathrm{e}} \\
\text { Number of mammogram providers in PCSA }\end{array}$ & $\begin{array}{l}8,677 \\
8,677 \\
7,717 \\
8,675 \\
8,150\end{array}$ & $\begin{array}{r}1.27 \\
7.69 \\
.29 \\
4.48 \\
27.0\end{array}$ & $\begin{array}{l}84.4 \\
315 . \\
38.8 \\
466 . \\
2,563\end{array}$ & $\begin{array}{l}1.15(1.09,1.22)^{* * *} \\
1.03(1.02,1.05)^{* * *} \\
0.83(0.75,0.92)^{* * *} \\
0.98(0.97,0.99)^{* * *} \\
1.02(1.01,1.03)^{* *}\end{array}$ \\
\hline & $\begin{array}{l}\text { Unweighted } \\
\text { frequency }\end{array}$ & $\begin{array}{l}\text { Weighted } \\
\% \text { sample }\end{array}$ & $\begin{array}{l}\% \text { Used } \\
\text { mammogram }\end{array}$ & $\begin{array}{l}\text { Crude OR } \\
(95 \% \text { CI })\end{array}$ \\
\hline $\begin{array}{l}\text { Poverty level }^{\mathrm{f}} \\
>30 \% \text { of PCSA lives under } 200 \% \text { FPL } \\
<30 \% \text { of PCSA lives under } 200 \% \text { FPL }\end{array}$ & $\begin{array}{l}4,943 \\
3,734\end{array}$ & $\begin{array}{l}52.4 \\
47.5\end{array}$ & $\begin{array}{l}64.0 \\
68.7\end{array}$ & $\begin{array}{c}0.81(0.73,0.90) \\
1.00^{* * *}\end{array}$ \\
\hline $\begin{array}{l}\text { PCSA density } \\
\text { Urban/suburban } \\
\text { Large/small town }\end{array}$ & $\begin{array}{l}7,011 \\
1,666\end{array}$ & $\begin{array}{l}80.7 \\
19.2\end{array}$ & $\begin{array}{l}67.3 \\
61.9\end{array}$ & $\begin{array}{c}1.27(1.09,1.47) \\
1.00^{* *}\end{array}$ \\
\hline $\begin{array}{l}\text { Race } \\
\text { Non-Hispanic white } \\
\text { Non-Hispanic black } \\
\text { Hispanic } \\
\text { Asian American }\end{array}$ & $\begin{array}{r}6,746 \\
1,333 \\
1,238 \\
238\end{array}$ & $\begin{array}{r}77.0 \\
11.0 \\
8.95 \\
2.98\end{array}$ & $\begin{array}{l}67.6 \\
64.8 \\
58.3 \\
53.9\end{array}$ & $\begin{array}{c}1.79(1.31,2.43) \\
1.58(1.13,2.2) \\
1.19(0.85,1.69) \\
1.00 * * *\end{array}$ \\
\hline $\begin{array}{l}\text { Marital status } \\
\text { Married } \\
\text { Unmarried (incl. LWP) }\end{array}$ & $\begin{array}{l}4,372 \\
5,278\end{array}$ & $\begin{array}{l}59.7 \\
40.2\end{array}$ & $\begin{array}{l}70.8 \\
59.0\end{array}$ & $\begin{array}{c}1.68(1.53,1.86) \\
1.00 * * *\end{array}$ \\
\hline $\begin{array}{l}\text { Family income } \\
\qquad 20,000 \\
\quad \$ 20,000-34,999 \\
\$ 35,000-54,999 \\
\$ 55,000-74,999 \\
\geq \$ 75,000\end{array}$ & $\begin{array}{r}2,594 \\
1,556 \\
1,380 \\
780 \\
1,605\end{array}$ & $\begin{array}{l}23.5 \\
18.3 \\
18.0 \\
11.7 \\
28.3\end{array}$ & $\begin{array}{l}50.9 \\
62.1 \\
67.5 \\
71.7 \\
77.4\end{array}$ & $\begin{array}{c}0.33(0.28,0.38) \\
0.52(0.44,0.6) \\
0.65(0.54,0.78) \\
0.74(0.58,0.95) \\
\quad 1.00 * * *\end{array}$ \\
\hline $\begin{array}{l}\text { Limitations } \\
\text { Limited in any way } \\
\text { Not limited in any way }\end{array}$ & $\begin{array}{l}4,853 \\
4,813\end{array}$ & $\begin{array}{l}48.2 \\
51.7\end{array}$ & $\begin{array}{l}64.3 \\
67.7\end{array}$ & $\begin{array}{c}0.86(0.78,0.95) \\
1.00^{* *}\end{array}$ \\
\hline $\begin{array}{l}\text { Health insurance } \\
\text { Not covered } \\
\text { Covered }\end{array}$ & $\begin{array}{l}1,054 \\
8,613\end{array}$ & $\begin{array}{l}9.93 \\
90.0\end{array}$ & $\begin{array}{l}37.3 \\
69.3\end{array}$ & $\begin{array}{c}0.26(0.23,0.31) \\
1.00^{* * *}\end{array}$ \\
\hline $\begin{array}{l}\text { Age group } \\
\quad 40-49 \\
50-64 \\
65+\end{array}$ & $\begin{array}{l}3,050 \\
3,607 \\
3,025\end{array}$ & $\begin{array}{l}34.5 \\
38.4 \\
27.0\end{array}$ & $\begin{array}{l}63.2 \\
71.2 \\
62.4\end{array}$ & $\begin{array}{l}1.04(0.92,1.17) \\
1.49(1.31,1.68) \\
1.00 * * *\end{array}$ \\
\hline $\begin{array}{l}\text { Education } \\
\text { Less than H.S. } \\
\text { H.S. grad } \\
\text { Some college } \\
\text { College grad }\end{array}$ & $\begin{array}{l}1,868 \\
2,970 \\
2,582 \\
2,191\end{array}$ & $\begin{array}{l}16.6 \\
31.8 \\
27.3 \\
24.1\end{array}$ & $\begin{array}{l}52.1 \\
64.3 \\
68.1 \\
76.3\end{array}$ & $\begin{array}{c}0.34(0.29,0.39) \\
0.56(0.49,0.64) \\
0.66(0.58,0.76) \\
1.00 * * *\end{array}$ \\
\hline $\begin{array}{l}\text { \#MD visits in last year } \\
\text { None } \\
1 \\
2-5 \\
6+\end{array}$ & $\begin{array}{l}1,002 \\
1,299 \\
4,090 \\
3,234\end{array}$ & $\begin{array}{l}10.1 \\
13.7 \\
43.1 \\
32.9\end{array}$ & $\begin{array}{l}26.8 \\
62.0 \\
72.0 \\
72.1\end{array}$ & $\begin{array}{c}0.14(0.12,0.17) \\
0.63(0.54,0.74) \\
0.99(0.88,1.12) \\
1.00 * * *\end{array}$ \\
\hline $\begin{array}{l}\text { Nativity } \\
\text { Born in USA } \\
\text { Born outside USA/US territories }\end{array}$ & $\begin{array}{l}8,246 \\
1,427\end{array}$ & $\begin{array}{l}87.0 \\
12.9\end{array}$ & $\begin{array}{l}66.8 \\
61.1\end{array}$ & $\begin{array}{c}1.28(1.11,1.48) \\
1.00 * * *\end{array}$ \\
\hline $\begin{array}{l}\text { Smoking status } \\
\text { Current } \\
\text { Former } \\
\text { Never }\end{array}$ & $\begin{array}{l}1,611 \\
2,293 \\
5,763\end{array}$ & $\begin{array}{l}16.4 \\
23.7 \\
59.7\end{array}$ & $\begin{array}{l}55.0 \\
70.6 \\
67.4\end{array}$ & $\begin{array}{c}0.59(0.52,0.67) \\
1.16(1.03,1.31) \\
1.00^{* * *}\end{array}$ \\
\hline $\begin{array}{l}\text { Employment status } \\
\text { Full-time } \\
\text { Not full-time }\end{array}$ & $\begin{array}{l}3,884 \\
5,798\end{array}$ & $\begin{array}{l}41.5 \\
58.4\end{array}$ & $\begin{array}{l}69.6 \\
63.5\end{array}$ & $\begin{array}{c}1.31(1.19,1.46) \\
1.00 * * *\end{array}$ \\
\hline $\begin{array}{l}\text { Health status } \\
\text { Excellent/very good } \\
\text { Fair/poor }\end{array}$ & $\begin{array}{l}7,797 \\
1,881\end{array}$ & $\begin{array}{l}82.5 \\
17.4\end{array}$ & $\begin{array}{l}67.7 \\
58.4\end{array}$ & $\begin{array}{c}1.50(1.32,1.7) \\
1.00^{* * *}\end{array}$ \\
\hline
\end{tabular}

${ }^{\mathrm{a}}$ The number of respondents by each characteristic may not add up to the total unweighted count due to missing values.

${ }^{\mathrm{b}} p$-Value calculated using Wald $F$ test: $p$-value: $* * \leq 0.01 ; * * * \leq 0.001$.

${ }^{c}$ Continuous variable.

${ }^{\mathrm{d}}$ IMG, international medical graduate; Ob-Gyns, obstetricians and gynecologists. Source: Goodman. ${ }^{23}$

${ }^{\mathrm{e}}$ Source: RTI Impact Factor Web Data.

${ }^{f}$ FPL, federal poverty line. Source: Goodman and Shipman. ${ }^{28}$ 
Table 3. Predictive Margin, Adjusted Odds Ratios, AND 95\% CI FOR RECOMMENDATION FOR MAMMOGRAPHY

\begin{tabular}{|c|c|c|}
\hline Characteristic $^{\mathrm{a}}$ & $\begin{array}{l}\text { Predictive } \\
\text { margin }\end{array}$ & $\begin{array}{l}\text { Adjusted } O R \\
\quad(95 \% C I)\end{array}$ \\
\hline $\begin{array}{l}\text { Number Ob-GyNs in PCSA } \\
\text { per } 10,000 \text { population }\end{array}$ & 0.63 & $1.09(1.02,1.16)^{* *}$ \\
\hline $\begin{array}{l}\text { Average distance to closest } \\
\text { mammogram provider } \\
\text { in PCSA }\end{array}$ & 0.63 & $0.98(0.97,0.99)^{*}$ \\
\hline \multicolumn{3}{|l|}{ Race } \\
\hline Non-Hispanic white & 0.63 & 1.00 \\
\hline Non-Hispanic black & 0.59 & $0.81(0.68,0.96)^{*}$ \\
\hline Hispanic & 0.63 & $0.99(0.79,1.24)$ \\
\hline Asian American & 0.51 & $0.59(0.41,0.84) * * *$ \\
\hline \multicolumn{3}{|l|}{ Marital status } \\
\hline Married & 0.64 & 1.00 \\
\hline Unmarried & 0.60 & $0.83(0.74,0.94) * * *$ \\
\hline \multicolumn{3}{|l|}{ Family income } \\
\hline$<20,000$ & 0.59 & $0.80(0.66,0.97)^{*}$ \\
\hline$\$ 20,000-34,999$ & 0.62 & $0.90(0.76,1.06)$ \\
\hline$\$ 35,000-54,999$ & 0.64 & 1.00 \\
\hline$\$ 55,000-74,999$ & 0.63 & $0.96(0.78,1.18)$ \\
\hline$\geq \$ 75,000$ & 0.64 & $1.01(0.84,1.22)$ \\
\hline \multicolumn{3}{|l|}{ Limitations } \\
\hline Limited in any way & 0.65 & $1.21(1.08,1.35)^{* * *}$ \\
\hline Not limited in any way & 0.60 & 1.00 \\
\hline \multicolumn{3}{|l|}{ Health insurance } \\
\hline Not covered & 0.52 & $0.61(0.50,0.74) * * *$ \\
\hline Covered & 0.63 & 1.00 \\
\hline \multicolumn{3}{|l|}{ Age group } \\
\hline $40-49$ & 0.62 & $1.13(0.96,1.34)$ \\
\hline $50-64$ & 0.66 & $1.36(1.18,1.58) * * *$ \\
\hline $65+$ & 0.59 & 1.00 \\
\hline \multicolumn{3}{|l|}{ \#MD visits in last year } \\
\hline None & 0.33 & 1.00 \\
\hline 1 & 0.60 & $3.09(2.41,3.95) * * *$ \\
\hline $2-5$ & 0.65 & $3.98(3.20,4.93) * * *$ \\
\hline $6+$ & 0.66 & $4.17(3.30,5.27)^{* * *}$ \\
\hline
\end{tabular}

$p$-Value: $*<0.05 ; * * \leq 0.01 ; * * * \leq 0.001$.

${ }^{a}$ Model was also adjusted for PCSA poverty level, nativity, education, region of the United States, and smoking status.

In Table 4, each one-unit increase in the number of Ob-GyNs per 10,000 population increased the odds of mammography use by 9\% (adjusted OR: $1.09,95 \%$ CI: $1.02-$ 1.16). Only $51 \%$ of Asian American women reported mammography use in the past 2 years, a rate that was $15 \%$ lower than the rate for non-Hispanic whites. Non-Hispanic black women had a $4 \%$ higher rate of mammography use than nonHispanic whites. Results showed comparatively lower rates of mammography use for women with less than a high school education $(61 \%)$, for women in poor health $(63 \%)$, for women residing in families with incomes of less than $\$ 20,000$ $(61 \%)$, and for women lacking health insurance coverage $(51 \%)$. Unmarried women $(63 \%)$, those in poor health $(63 \%)$, women with functional limitations $(66 \%)$, and current smokers $(62 \%)$ were also less likely to report having used mammography. Rates of mammography use were positively associated with seeing a doctor one or more times during the past year $(61 \%-74 \%)$, being a college graduate $(72 \%)$, residing in a family with $\$ 75,000$ or more in income $(71 \%)$, and being aged
Table 4. Predictive Margin, Adjusted Odds Ratios, AND 95\% CI FOR MAMMOgRAPHY UsE

\begin{tabular}{|c|c|c|}
\hline Characteristic $^{\mathrm{a}}$ & $\begin{array}{l}\text { Predictive } \\
\text { Margin }\end{array}$ & $\begin{array}{l}\text { Adjusted } O R \\
(95 \% \text { CI })\end{array}$ \\
\hline $\begin{array}{l}\text { Number } \mathrm{Ob}-\mathrm{GyNs} \\
\quad \text { per } 10,000 \text { population }\end{array}$ & 0.67 & $1.09(1.02,1.16)^{* *}$ \\
\hline \multicolumn{3}{|l|}{ Race } \\
\hline Non-Hispanic white & 0.67 & 1.00 \\
\hline Non-Hispanic black & 0.71 & $1.26(1.05,1.51)^{*}$ \\
\hline Hispanic & 0.67 & $1.02(0.80,1.29)$ \\
\hline Asian American & 0.51 & $0.47(0.31,0.71)^{* * *}$ \\
\hline \multicolumn{3}{|l|}{ Marital status } \\
\hline Married & 0.70 & 1.00 \\
\hline Unmarried & 0.63 & $0.71(0.62,0.82)^{* * *}$ \\
\hline \multicolumn{3}{|l|}{ Education } \\
\hline Less than H.S. & 0.61 & $0.74(0.61,0.89)^{* * *}$ \\
\hline H.S. grad & 0.67 & 1.00 \\
\hline Some college & 0.68 & $1.00(0.85,1.18)$ \\
\hline College grad & 0.72 & $1.34(1.14,1.58)^{* * *}$ \\
\hline \multicolumn{3}{|l|}{ Limitations } \\
\hline Limited in any way & 0.66 & $0.87(0.76,0.99)^{*}$ \\
\hline Not limited in any way & 0.68 & 1.00 \\
\hline \multicolumn{3}{|l|}{ Health status } \\
\hline Fair/poor & 0.63 & $0.76(0.63,0.91)^{* * *} *$ \\
\hline Excellent/very good & 0.68 & 1.00 \\
\hline \multicolumn{3}{|l|}{ Family income } \\
\hline$<20,000$ & 0.61 & $0.75(0.62,0.91)^{* * *}$ \\
\hline$\$ 20,000-34,999$ & 0.68 & $1.06(0.88,1.26)$ \\
\hline$\$ 35,000-54,999$ & 0.67 & 1.00 \\
\hline$\$ 55,000-74,999$ & 0.68 & $1.05(0.85,1.31)$ \\
\hline$\geq \$ 75,000$ & 0.71 & $1.23(1.01,1.49)^{*}$ \\
\hline \multicolumn{3}{|l|}{ Health insurance } \\
\hline Not covered & 0.51 & $0.42(0.34,0.51)^{* * *}$ \\
\hline Covered & 0.69 & 1.00 \\
\hline \multicolumn{3}{|l|}{ Age group } \\
\hline $40-49$ & 0.63 & $0.91(0.76,1.09)$ \\
\hline $50-64$ & 0.71 & $1.41(1.20,1.67) * * *$ \\
\hline $65+$ & 0.65 & 1.00 \\
\hline \multicolumn{3}{|l|}{ Smoking status } \\
\hline Current & 0.62 & $0.74(0.63,0.86)^{* * *}$ \\
\hline Former & 0.68 & $1.04(0.91,1.20)$ \\
\hline Never & 0.68 & 1.00 \\
\hline \multicolumn{3}{|c|}{ Number MD visits in last year } \\
\hline None & 0.31 & 1.00 \\
\hline 1 & 0.61 & $3.94(3.06,5.08) * * *$ \\
\hline $2-5$ & 0.71 & $6.42(5.14,8.01) * * *$ \\
\hline $6+$ & 0.75 & $8.16(6.30,10.57)^{* * *}$ \\
\hline
\end{tabular}

$p$-Value: $*<0.05 ; * * \leq 0.01 ; * * * \leq 0.001$

${ }^{a}$ Model was also adjusted for nativity, employment status, PCSA poverty level, PCSA density, average distance to closest mammography provider in PCSA, and number of mammogram providers in PCSA.

50-64 years (71\%). PCSA distance to closest mammography provider, poverty level, number of mammogram providers and population density, nativity, or employment status were no longer significant in the adjusted mammography use results.

Table 5 shows the results from the adjusted analyses using the alternative physician supply measures listed in Tables 1 and 2 . The $p$-value for the indicator of primary care doctors was statistically significant (at 0.02 ) in both the recommendation 
Table 5. Predictive Margin, Adjusted Odds Ratios, and 95\% CI for Mammography ReCOMMENDATION OR MAMMOgRaPhy UsE

\begin{tabular}{|c|c|c|c|c|}
\hline \multirow[b]{2}{*}{ PCSA physician supply measure } & \multicolumn{2}{|c|}{ Mammography recommendation ${ }^{\mathrm{a}}$} & \multicolumn{2}{|c|}{ Mammography use } \\
\hline & $\begin{array}{l}\text { Predicted } \\
\text { margin }\end{array}$ & $\begin{array}{l}\text { Adjusted } \\
\text { OR }\end{array}$ & $\begin{array}{l}\text { Predicted } \\
\text { margin }\end{array}$ & $\begin{array}{l}\text { Adjusted } \\
\text { OR }\end{array}$ \\
\hline Number of PCMD in PCSA per 10,00 & 0.6 & $1.02(1.00-1.0$ & 0 . & $1.02(1.00-1.04)^{*}$ \\
\hline Ratio of IMG Ob-GyN To US Ob-GyN & NS & - & 0.67 & $0.82(0.73,0.92)^{*}$ \\
\hline
\end{tabular}

$p$-Value: $*<0.05 ; * * * \leq 0.001$.

${ }^{a}$ Model was adjusted for race, marital status, family income, limitations, health insurance, age group, number of MD visits, nativity, education, region, smoking status, PCSA poverty level, and distance to mammography provider in PCSA.

${ }^{\mathrm{b}}$ Model was adjusted for race, marital status, education, family income, limitations, health status, health insurance, age group, number of MD visits, nativity, employment status, PCSA poverty level, PCSA density, distance to mammography provider in PCSA, and number of mammogram providers in PCSA.

receipt and mammography test results. However, the confidence intervals contained 1 . A one-unit increase in the ratio of IMG Ob-GyNs to US Ob-GyNs decreased the odds of mammography use (OR: 0.83; 95\% CI: 0.74-0.93). The indicator for FTEPCPs was no longer significantly associated with recommendation receipt in the adjusted analysis.

\section{Discussion}

This study is the first to investigate how medical market characteristics, measured at the PCSA level, affect rates of recommendation receipt and screening mammography use among a national sample of women. Most importantly, unlike in previous investigations, where the available data dictated the level of geography at which the physician supply indicator was measured, this study was able to fully examine the impact of physician density in medical markets where respondents actually resided and likely received their outpatient care. $^{32,33}$

The findings suggest support for the hypothesis that PCSAs with higher numbers of primary care providers may improve access to and use of preventive breast cancer services. In the multivariable analysis, the supply of OB-GyNs was positively associated with both recommendation receipt and mammography use. However, the general measure for the supply of primary care physicians was not significantly associated with either outcome measure. Since the mean number of primary care physicians per 10,000 population (7.75, SD: 319.5$)$ was six times greater than the mean number of Ob-GyNs (1.29, SD: 85.4), this result was unexpected. However, patients of $\mathrm{Ob}-\mathrm{GyNs}$ have higher recorded rates of screening mammography, and Ob-GyNs are more likely to provide recommendations for mammography when compared with other primary care physicians. ${ }^{34}$ Additionally, relative to other specialties, Ob-GyNs were less likely to not recommend mammography when patients reach a certain age. ${ }^{28}$

Before the inclusion of the distance to closest mammography variable in the recommendation receipt model, the categorical measure of FTEPCPs (Table 1) and the indicator for all primary care physicians were both significant. Since distance to a mammography provider was an important predictor in this model's adjusted results, it may have mediated the effect of physician density on recommendation receipt. In addition, the inclusion in the mammogram use model of the number of mammography providers and the average distance to providers attenuated the effect of the primary care physician supply measure.

Women were less likely to report having a mammogram in the past 2 years if they resided in PCSAs with a higher ratio of IMG to non-IMG Ob-GyNs. Previous research indicates that IMGs as a group were more likely to practice in underserved areas and in locales characterized by rural populations in poverty, ${ }^{35,36}$ and unlike in other studies, area-level poverty or rural/urban distinctions were not determinants for mammography use in the adjusted analyses. ${ }^{19,37}$ In addition, as these factors were controlled for in the model, it is not likely that rural/urban distinctions or residence in poverty areas can explain the IMG Ob-GyN finding.

A federal government study showed that patients who relied on Medicaid or other public insurance coverage were more likely to see IMG physicians as a group in 2005-2006. ${ }^{38}$ Since the health insurance variable used in this study did not distinguish the type of respondent's health insurance coverage, we cannot know if this played a part in determining the sign of the coefficient estimate on the IMG Ob-GyN variable.

In results from this study, not shown, but available from the author, the number of mammography providers was significantly associated with mammography use (OR: 1.23; 95\% CI: 1.02-1.49) only after a control was made for the supply of IMG primary care doctors. The supply of IMG primary care doctors was itself not significant. Further research is needed to derive accurate conclusions. Different types of primary care physician density indicators (i.e., IMG physicians) should be included in studies on geographic access to mammography and its relationship to breast cancer screening. ${ }^{39}$ More research on where IMG Ob-GyNs are located throughout the United States as well as on the populations they serve could yield insight into the association between locales where IMG Ob-GyNs practice and women's use of screening mammography.

Overall, PCSA indicators were less powerful predictors of both recommendation receipt and mammography use when set side by side with many of the individual-level measures tested. Past studies that were reliant on contextual factors estimated at the county or census tract level have also found these measures to be less powerful for explaining mammography use variations. ${ }^{18,19,40}$ From a comparative perspective, the inclusion of PCSA information in the empirical tests did not greatly alter the strength of key individual factors commonly reported in similar studies on mammography utilization. ${ }^{7,32}$ Asian Americans, the unmarried, those residing 
in families with low/poverty-level incomes, and lacking health insurance coverage were less likely to report either receipt of a doctor's recommendation or use of mammography.

The results should be interpreted in light of a number of limitations. Self-reports of screening use and recommendation receipt can be inaccurate due to recall bias or the overestimating of adherence. ${ }^{41}$ The cross-sectional associations between physician capacity and getting a doctor's recommendation, or using screening mammography, do not establish directionality or causality. Variables included in the tests, such as age, income, education, insurance coverage, and number of doctor visits, attempted to capture components of individual-level demand for preventive breast cancer services. Still, the focus of this study was placed squarely on the supply-side characteristics of the primary care workforce. The empirical tests did not include a control for residential segregation in the PCSA by race/ ethnicity. Yet, residential segregation by race/ethnicity has been associated with locales characterized by shortages of primary care providers. $^{42,43}$

A further qualification concerns the construction of the physician capacity variables from data found in the 2005-2006 American Medical Association Physician Masterfile. Pediatric doctors are incorporated into the primary care physician indicators and their inclusion may dilute the expected relationship between physician density and the outcome measures. Moreover, the Masterfiles are generally in need of careful updating as they include physicians who have retired, while excluding newer physicians and their specialties. ${ }^{44,45}$ Yet, there are numerous advantages to using PCSA data for investigating the infrastructure and composition of medical markets in the United States. Researchers can access more recent data online and link PCSA information to other cancer-related survey data to investigate cancer screening use as well as other outcomes. ${ }^{46}$

\section{Conclusion}

This study shows a statistically significant association between the supply of Ob-GyNs and rates of mammography recommendation receipt and mammogram use in the United States. The associations were positive when considering the supply of Ob-GyNs in general. However, for reported mammogram use, the association was negative in PCSAs where the ratio of IMG Ob-GyNs to US-trained Ob-GyNs was high. Prioritizing a more equitable distribution of the supply of primary care providers is essential as 7 million Americans were estimated to have lived in PCSAs where the demand for primary care providers would likely exceed supply by more than $10 \%$ because of the Affordable Care Act's (ACA) expansion of insurance coverage. ${ }^{47}$

The ACA contains provisions (if properly implemented and funded) that would positively affect not only the size but also the geographic location of the primary care workforce in the United States. ${ }^{48}$ This is critical as there are geographic imbalances in the current supply of ob-gyn physicians at a time when the demand for women's health services is forecasted to grow. ${ }^{49}$ Since the ACA requires insurance plans to offer no-cost coverage for mammograms, a policy that focused on reducing national access disparities to primary care physicians in general and to ob-gyn physicians in particular, could effectively promote greater use of breast health services by age-appropriate women.

\section{Acknowledgments}

I would like to acknowledge William Waldron of Applied Statistical Programming for using his formidable research skills and ingenuity to merge and analyze the data set used for this paper. Also, the encouragement and support of Negasi Beyene, of the Research Data Center, National Center for Health Statistics.

\section{Author Disclosure Statement}

No competing financial interests exist.

\section{References}

1. Mandelblatt JS, Yabroff KR. Equitable access to cancer services: A review of barriers to quality care. Cancer 1999; 86:2378-2390.

2. Taplin SH, Ichikawa L. Evaluating organized breast cancer screening implementation: The prevention of late-stage disease? Cancer Epidem Biomar Prev 2004;13:225-234.

3. Schonberg MA, McCarthy EP. Factors influencing elderly women's mammography screening decisions: Implications for counseling. BMC Geriatr 2007;7:26.

4. González P, Borrayo EA. The role of physician involvement in Latinas' mammography screening adherence. Womens Health Iss 2011;21:165-170.

5. Alexandraki I, Mooradian AD. Barriers related to mammography use for breast cancer screening among minority women. J Natl Med Assoc 2010;102:206-218.

6. Hawley ST, Earp JA. The role of physician recommendation in women's mammography use: Is it a 2-stage process? Med Care 2000;38:392-403.

7. Schueler KM, Chu PW. Factors associated with mammography utilization: A systematic quantitative review of the literature. J Womens Health 2008;17:1477-1498.

8. Lange F. The role of education in complex health decisions: Evidence from cancer screening. J Health Econ 2011;30:43-54.

9. O'Malley MS, Hawley EJ. The association of race/ethnicity, socioeconomic status, and physician recommendation for mammography: Who gets the message about breast cancer screening? Am J Public Health 2001;91:49-54.

10. Dominick KL, Skinner CS. Provider characteristics and mammography recommendation among women in their 40s and 50s. J Womens Health 2003;12:61-71.

11. Bazargan M, Bazargan SH. Mammography screening and breast self-examination among minority women in public housing projects: The impact of physician recommendation. Cell Mol Biol (Noisy-le-grand) 2003;49:1213-1218.

12. Roetzheim RG, Ferrante JM, Lee JH. Influence of primary care on breast cancer outcomes among Medicare beneficiaries. Ann Fam Med 2012;10:401-411.

13. Akinyemiju TF, Soliman AS. Healthcare access and mammography screening in Michigan: A multilevel crosssectional study. Int J Equity in Health 2012;11:16-26.

14. Gorey KM, Luginaah IN. The supply of physicians and care for breast cancer in Ontario and California, 1998 to 2006. Can J Rural Med 2011;16:47-54.

15. Sundmacher L, Busse R. The impact of physician supply on avoidable cancer deaths in Germany: A spatial analysis. Health Pol 2011;103:53-62.

16. Pornet C, Dejardin O. Socioeconomic and healthcare supply statistical determinants of compliance to mammography screening programs: A multilevel analysis in Calvados, France. Cancer Epidem 2010;34:309-315. 
17. Benjamins MR, Kirby JB. County characteristics and racial and ethnic disparities in the use of preventive services. Prev Med 2004;39:704-712.

18. Litaker D, Tomolo A. Association of contextual factors and breast cancer screening: Finding new targets to promote early detection. J Womens Health 2007;16:36-45.

19. Coughlin SS, Leadbetter S. Contextual analysis of breast and cervical cancer screening and factors associated with health care access among United States women. Soc Sci Med 2008;66:260-275.

20. Ferrante JM, Gonzalez EC. Effects of physician supply on early detection of breast cancer. J Am Board Fam Pract 2000;13:408-414.

21. Barry J, Breen N. Significance of increasing poverty levels for determining late-stage breast cancer diagnosis in 1990 and 2000. J Urb Health 2012;89:614-627.

22. Butler DC, Petterson S. Measures of social deprivation that predict health care access and need within a rational area of primary care service delivery. Health Serv Res 2012;48: 539-559.

23. Goodman D. HRSA AMA data dictionary 1008 (REVISED) Version date: October 21, 2008. Print Date: June 3, 2009. Prepared for the Health Resources and Services Administration under contract modification number 25002-0013.

24. National Center for Health Statistics. Data File Documentation, National Health Interview Survey. 2005 (Machine Readable Data File and Documentation). Maryland: National Center for Health Statistics, Centers for Disease Control and Prevention Hyattsville; 2006.

25. Smith R, Cokkinides V. Cancer screening in the United States, 2007: A review of current guidelines, practices, and prospects. CA Cancer J Clin 2007;57:90-104.

26. Han PK, Klabunde CN, Breen N. Multiple clinical practice guidelines for breast and cervical cancer screening: Perceptions of US primary care physicians. Med Care 2011; 49:139-148.

27. American Cancer Society. American Cancer Society guidelines for the early detection of cancer [Internet]. Atlanta (GA): ACS. Available at: www.cancer.org/healthy/ findcancerearly/cancerscreeningguidelines/american-cancersociety-guidelines-for-the-early-detection-of-cancer Accessed July 6, 2016.

28. Goodman D, Shipman S. Primary Care Service Area identification of new access points for community health centers. Version date: November 18, 2007. Print Date: November 18, 2007. Prepared for the Health Resources and Services Administration under contract modification number $\mathrm{HHSH} 230$ 200732007C.

29. RTI Spatial Impact Factor Web Data. [database online] Available at: https://rtispatialdata.rti.org Accessed July 6, 2016.

30. SUDAAN, Version 10.0.1. [computer program]. Research Triangle Institute, Research Triangle Park, NC.

31. Carle AC. Fitting multilevel models in complex survey data with design weights: Recommendations. BMC Med Res Methodol 2009;9:49.

32. Dailey AB, Brumback BA, Livingston MD. Area-level socioeconomic position and repeat mammography screening use: Results from the 2005 National Health Interview Survey. Cancer Epidem Biomar Prev 2011;20:2331-2344.

33. Mazumdar S, Butler D, Baheri N. How useful are Primary Car Service Areas? Evaluating PCSAs as a tool for measuring Primary Care Practitioner access. App Geogr 2016; $72: 47-54$.
34. Bhosle M, Samuel S. Physician and patient characteristics associated with outpatient breast cancer screening recommendations in the United States: Analysis of the National Ambulatory Medical Care Survey Data 1996-2004. Breast Cancer Res Treat 2007;103:53-59.

35. Thompson MJ, Hagopian A. Do International medical graduates (IMGs) "Fill the Gap" in rural primary care in the United States? A national study. J Rural Health 2009; 25:124-134.

36. Xierali IM, Rinaldo JC. Family physician participation in maintenance of certification. Ann Fam Med 2011;9:203-210.

37. Khan N, Kaestner R. Does supply influence mammography screening? Am J Health Behav 2010;34:465-475.

38. Hing E, Lin S. Role of international medical graduates providing office-based medical care: United States, 20052006. Centers for Disease Control and Prevention, February 2009. Available at: www.cdc.gov/nchs/data/databriefs/ db13.pdf Accessed July 6, 2016.

39. Khan-Gates J, Ersek J, Eberth J. Geographic access to mammography and its relationship to breast cancer screening and stage at diagnosis: A systematic review. Womens Health Iss 2015;25:482-493.

40. Pathman D, Ricketts T. How adults' access to outpatient physician services relates to the local supply of primary care physicians in the rural southeast. Health Serv Res 2006:41:79-102.

41. Armstrong K, Long J, Shea J. Measuring adherence to mammography screening recommendations among lowincome women. Prev Med 2004;38:754-760.

42. White K, Haas JS. Elucidating the role of place in health care disparities: The example of racial/ethnic residential segregation. Health Serv Res 2012;47:1278-1299.

43. Gaskin DJ, Dinwiddie GY. Residential segregation and the availability of primary care physicians. Health Serv Res 2012;47:2353-2376.

44. Chang $\mathrm{CH}$, Stukel TA. Primary care physician workforce and Medicare beneficiaries' health outcomes. JAMA 2011;305:2096-2104.

45. Staiger DO, Auerbach DI. Comparison of physician workforce estimates and supply projections. JAMA 2009;302: 1674-1680.

46. Health Resources and Services Administration. Health Workforce, Primary Care Services Area. [database online]. Available at: http://bhpr.hrsa.gov/healthworkforce/data/ primarycareserviceareas/index.html Accessed July 6, 2016.

47. Huang ES, Finegold K. Seven million Americans live in areas where demand for primary care may exceed supply by more than 10 percent. Health Aff 2013;20:10-377.

48. Heisler EJ. Physician supply and the Affordable Care Act. Congressional Research Service 2013. 7-5700 www.crs .gov R42029. Available at: http://healthcarereform.procon .org/sourcefiles/crs-physician-supply-and-affordable-careact.pdf Accessed July 6, 2016.

49. Dall T, Chakrabarti R, Storum M. Estimated demand for women's health services by 2020. J Womens Health 2013; 22:643-648.

Address correspondence to: Janis Barry, PhD

Department of Economics Fordham University 113 West 60th Street New York, NY 10023

E-mail: barryfiguero@fordham.edu 